\title{
Preface to the special issue on the crustal and upper mantle structure and geodynamics in Chinese mainland
}

\author{
Chunyong Wang \\ Institute of Geophysics, China Earthquake Administration, Beijing 100081, China
}

Over the past decade, one important development was the rapid and significant improvement of instruments and observation techniques, such as the broadband seismometers and the seismic-array observations, which permit to widespread collect seismic wave and then clearly demonstrate the crustal and upper mantle structure. The structural and tectonic settings are mainly obtained in the comprehensive analyses of the data from geology, geophysics and seismological phenomena. China is situated at the junction of the eastern Eurasia plate, India plate and Pacific plate. It is a region, where the tectonic movements are active, and the continental earthquakes with large magnitude frequently occur. Therefore, Chinese seismologists pay more attention to the seismotectonics and the continental dynamics. This requires a comprehensive understanding of crustal and upper mantle structures related to the plate tectonics and the mantle dynamics. This issue collected 15 papers devoting to these goals, although it is merely a small part of the research results in China.

Deep Seismic Sounding (DSS) is an important technique of exploring the velocity structure in crust and upper mantle. The goal is to understand the crustal geodynamic process and seismogenic tectonic settings. The DSS profiles with a total length of over $10000 \mathrm{~km}$ were carried out in China in the last thirty years. The 2-D crustal structures were determined based on the data collected along the profiles. On the basis of previous studies, Xiankang Zhang et al summarized the crustal velocity structures and their tectonic implications in different tectonic-block regions of the Chinese mainland, presented the typical velocity structures and average crustal velocities in different tectonic blocks, and discussed their tectonic implications. Chunyong Wang et al, Zhongjie Zhang et al, Fuyun Wang and Xiankang Zhang,
Lei Yang et al respectively present their results of 2-D crustal structure along deep seismic sounding profile by use of different interpretive methods. Rizheng He et al carried out the integrated geophysical investigation along the Western Kunlun-Tarim-Tianshan geosciences transect, and present a new pattern of continent-continental collision beneath the Western Kunlun, Northwestern China. Hongwei Zheng et al studied the transverse density structure along the Daagze-DeqenDuoma profile using a joint gravity-seismic inversion technique. Qiusheng Li et al present that large explosions may be a useful exploration tool in the Tibetan plateau.

Magnetotelluric sounding (MT) is used to explore the electricity structure of the crust and upper mantle. Guoze Zhao et al studied the electromagnetic anomaly before earthquakes measured by electromagnetic experiments, in which anomalous variations of resistivity and electromagnetic fields were recorded although it is only an experiment carried out with purpose to test the possibility of electromagnetic methods for earthquake monitoring.

Seismic network observation is one of the methods of determining 3-D velocity structure of the crust and upper mantle. Zhifeng Ding et al carried out the tomographic imaging of P-wave velocity structure beneath the region around Beijing by use of the $\mathrm{P}$ wave arrival times from local and teleseismic events. Jianping $\mathrm{Wu}$ et al studied S-wave velocity structure beneath Changbaishan volcano inferred from teleseismic receiver function. Songlin Li et al studied the fault-zone structures of northern and southern segments of the main central fault generated by $M_{\mathrm{S}} 8.0$ Wenchuan earthquake using fault zone trapped waves. Qingju Wu et al present a wavelet transformation method to measure interstation phase 
velocity.

Rongshan $\mathrm{Fu}$ et al studied the pervasion degree of convective mixing and investigations of mantle mixing. The results show that at the initial stage though the convective mixing pervasions are very different from mode to mode, after running for some time, the convective mixing pervasions for most modes are going to be a constant and the tracers are more evenly distributed in the whole mantle. Jianqiao Xu and Heping Sun studied the temporal variations in free core nutation $(\mathrm{FCN})$ period by using the tidal gravity observations of 18 -year duration recorded continuously with a superconducting gravimeter (SG) at Brussels. The results indicate that there exist decade-scale variations in the $\mathrm{FCN}$ period.

The editors would like to express their thanks to the authors for their willingness to share their results with the Earthquake Science. 